\title{
Minor Mergers and the Formation of Shell Galaxies
}

\author{
V. Charmandaris and F. Combes \\ Observatoire de Paris, DEMIRM, 61 Ave. de l'Observatoire, F-75014, \\ Paris, France
}

\begin{abstract}
We present a new mechanism which can explain the observed radial displacement between the stellar and gaseous shells observed around early type galaxies. Our model is based on the well-known phasewrapping process taking into account that a fraction of the interstellar medium is clumpy and consequently it experiences a low dynamical friction during a merging event. Moreover, our recent detection of cold molecular gas associated with the outer shells of Centaurus $\mathrm{A}$ is in agreement with the proposed model. This suggests that a considerable fraction of molecular gas in minor mergers may be distributed away from the nuclear regions and remain undetected.
\end{abstract}

\section{Introduction}

It is widely accepted that galaxy interactions and mergers have profound consequences to the morphology and evolution of galaxy groups. There have been numerous observational evidence suggesting that nearly every galaxy has undergone an interaction with a companion and that the accretion of small companions into more massive group members is a frequent event.

A typical signature of such a merger is the presence of optical shells or ripples around massive early type galaxies. Simulations of the stellar component have shown that the shells or ripples are created either by "phase-wrapping" of debris on nearly radial orbits (Quinn 1984 , Dupraz \& Combes 1986, 1987) or sometimes by "spatial-wrapping" of matter in thin disks (Hernquist \& Quinn 1989). Stars, modeled as collisionless particles, which were originally bound to the merging companions are liberated and oscillate with different periods around the new common central potential. Before they relax and due to their different periods of oscillation, they accumulate near the apocenters of their orbits, to form shell-like features.

The fact that this scenario is indeed occurring had been questioned by the recent observations of neutral hydrogen in systems containing shells. Schiminovich et al $(1994,1995)$ and Petric et al (1997) have detected diffuse $\mathrm{H}_{\mathrm{I}}$ gas shells displaced by approximately $1 \mathrm{kpc}$ just beyond the stellar ripples of Centaurus A, NGC 2865 and NGC 1210 . These observational results, which were followed by similar $\mathrm{H}$ I detections in other galaxies (van Gorkom, private communication) were a priori surprising. The reason was that we believe that shells are phase-wrapped stellar structures and that the diffuse gaseous and stellar components do not have the same behavior when approaching the center of the potential well, in quasi-radial orbits. Since the gas is more dissipative than the stars, numerical simulations suggested that it should always fall towards the center and therefore no gaseous shells should exist (Weil \& Hernquist 1993). 


\section{Our model}

However, the details of the gas dynamics in simulations depend strongly on the specific model adopted for the interstellar medium, and modeling of the gas using smoothed particle hydrodynamics (implying a lot of dissipation) may not be very appropriate. Kojima \& Noguchi (1997) carried out simulations of a disk satellite sinking into an elliptical using a sticky particle code and star formation, and revealed no segregation between stars and gas.
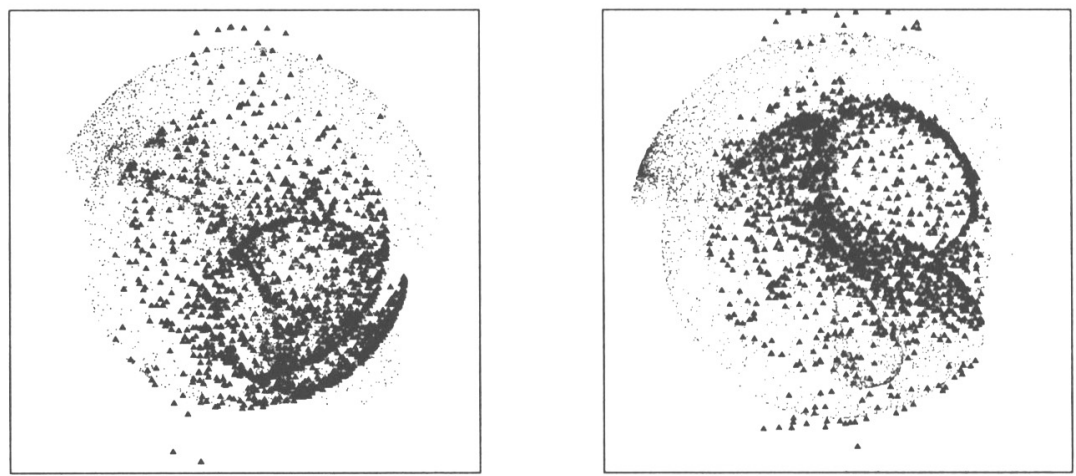

Figure 1. Simulations of the infall of a small spiral satellite into a giant elliptical, with low dissipation gas, and dynamical friction taken into account. The stars are black triangles, and gas clouds, dots.

We also believe that within the gas component in galaxies there exist a population of small and dense gas clouds, which have low dissipation. The dynamical behavior of those clouds would be intermediate between the stars and the diffuse $\mathrm{H}$ I gas and while most gas would end up in the center after the merger a fraction of it could remain in large distances from the nucleus forming the shells (see Fig. 1). To explain the spatial displacement between the gas and stars we consider that the radial scale length of the gaseous disks extends $\sim 1.5$ -2 times further than the stellar one. Hence, gas is less bound and it is liberated early from the companion by the tidal forces, while the stars are liberated later on (Combes \& Charmandaris 1999). Through dynamical friction, the stars have therefore time to lose a lot of energy, contrary to the gas. Dynamical friction also explains the dynamical range of the shell radii (Dupraz \& Combes 1987).

\section{Observations}

An observational test to our model would be the detection of the cold dense gas clouds in shells. Since these dense clouds should be in $\mathrm{H}_{2}$ form, we performed millimeter wave observations in a sample of shell galaxies, to look for molecular gas in shells via the $\mathrm{CO}(1-0)$ and $\mathrm{CO}(2-1)$ lines. The observations were carried out with the IRAM 30-m and the SEST 15-m telescopes on January and May 1999. The angular size of the telescope beams (22" and 44" at CO(1-0) line respectively) in conjunction with the small angular separation of the nucleus from 
the shells in distant galaxies made the detection of $\mathrm{CO}$ and the interpretation of the results very challenging.

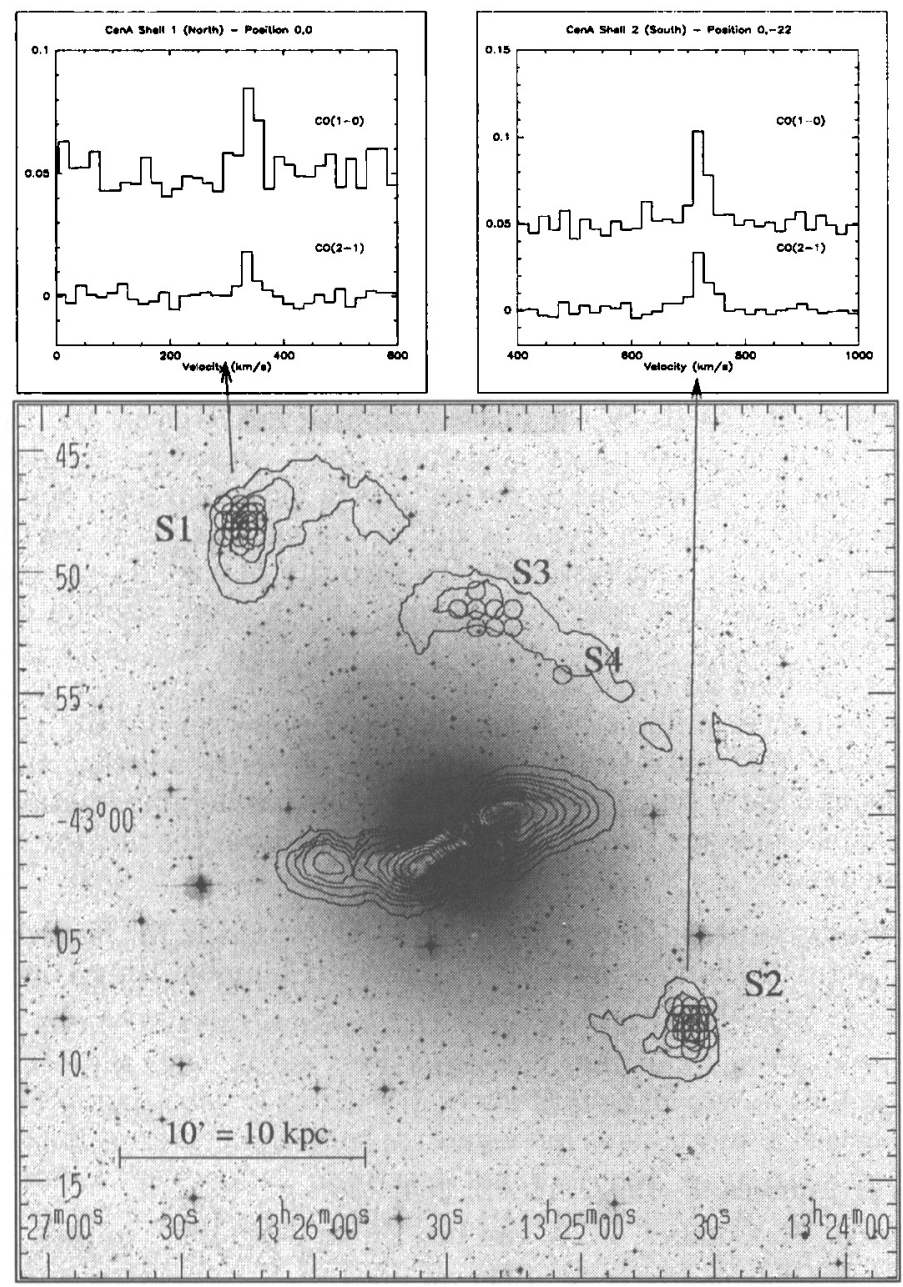

Figure 2. An optical DSS image of Centaurus A with the contours of the H I gas (from Schiminovich et al 1994) superimposed on it. The regions mapped in CO are presented in small circles (of the SEST beam size). Note the strong detections of molecular gas in the northern and southern shells.

\section{Results and Discussion}

Our IRAM observing run resulted in possible detection of $\mathrm{CO}$ in the southern shell of NGC 3656. However, our best and most surprising result was the discovery (see Fig. 2) of $\mathrm{CO}$ in the shells of Centaurus-A at a distance of $15 \mathrm{kpc}$ from the center of the elliptical galaxy (Charmandaris, Combes, van der Hulst 1999). 
Having mapped only a fraction of the shells of CenA in CO, we clearly detect $1.7 \times 10^{7} M_{\odot}$ of $\mathrm{H}_{2}$ in the northern shell (S1) and $2.2 \times 10^{7} M_{\odot}$ in the southern one (S2). The $\mathrm{CO}(2-1) / \mathrm{CO}(1-0)$ ratio is $\sim 0.7$ not much lower than the 0.9 observed in the nuclei of nearby spirals (Braine \& Combes 1992), and slightly above what is found in disks.

However, even though the $\mathrm{H} \mathrm{I}$ column density is very small in the shells the ratio of $\mathrm{HI}$ to $\mathrm{H}_{2}$ gas in the area of the shells we mapped is nearly unity, similar to the values found in the nuclear region of the galaxy. This is very surprising since the shells are in the outer parts of an elliptical galaxy. Moreover, the line width of the CO spectra in several pointing is less than $18 \mathrm{~km} \mathrm{~s}^{-1}$ resolution of our smoothed spectrum, and its velocities are in good agreement with those of the nearby $\mathrm{H}$ I gas.

The above result is compatible with the view that the dense clumps have been dragged in the shells by the phase-wrapping mechanism, and that the $\mathrm{H} \mathrm{I}$ diffuse gas has been photo-dissociated from there. Based on the detected $\mathrm{CO}$ emission at least $50 \%$ of the gas in the shells is in molecular form and more than $10 \%$ of the molecular gas in CenA is not in the nucleus. It is worth stressing that most probably we underestimate the quantity of cold $\mathrm{H}_{2}$ gas since due to limited telescope time, we were able to map only a small fraction of the area. Moreover, even though the gas clouds originate from the outer parts of the disrupted companion no corrections for low metallicity have been made.

The advances in millimeter astronomy and more specifically the development of ALMA will make technically feasible to verify whether this trend is observed in other early type galaxies. If so then a considerable fraction of molecular gas in minor mergers may be distributed away from the nuclear regions and remain undetected.

Acknowledgments. The authors would like to thank M. Noguchi (Tohoku Univ.) for useful discussions and J. van Gorkom (Columbia Univ.) for providing the H I data of Centaurus A.

\section{References}

Braine, J., \& Combes, F. 1992, A\&AS, 264, 433

Charmandaris, V., Combes, F., \& van der Hulst, J-M. 1999, in prep

Combes, F., \& Charmandaris, V. 1999, in prep

Dupraz C., \& Combes, F. 1986, A\&AS, 166, 53

Dupraz C., \& Combes, F. 1987, A\&AS, 185, L1

Hernquist, L., \& Quinn, P. 1989, ApJ, 342, 1

Kojima, M., \& Noguchi, M. 1997, ApJ, 481, 132

Petric, A., et al. 1997, BAAS, 191, 8212

Quinn, P. J. 1984, ApJ, 279, 596

Schiminovich, D., et al. 1994, ApJ, 423, L101

Schiminovich, D., et al. 1995, ApJ, 444, L77

Weil, M. L., \& Hernquist, L. 1993, ApJ, 405, 142 